\title{
AVALIAÇÃO DE IMPACTOS, PASSIVOS E DANOS AMBIENTAIS EM ZONAS COSTEIRAS DO BRASIL: REGIÃO DO LITORAL NORTE FLUMINENSE
}

\author{
Victor Guida de Freitas \\ Aluno de Ciências Biológicas \\ Meio Ambiente e Biodiversidade (UERJ) \\ $\triangle$ vgfreitas.92@gmail.com \\ Josimar Ribeiro de Almeida \\ Doutor - Professor Universidade do Estado do Rio de Janeiro (UERJ) - Pesquisador Senior Centro de \\ Engenharia Nuclear/USP - RJ - Brasil \\ Flávia Constantino da Vitória \\ Bióloga, Especialista em Gestão Ambiental e pesquisadora independente.
}

\begin{abstract}
Resumo:
A exploração petrolífera trouxe avanços tecnológicos e elevados ganhos financeiros de forma direta e indireta para municípios de sediam empresas desse ramo e trabalhadores de diversas áreas, movimentando a economia local, estadual e nacional, mas em contrapartida deste avanço surgiu o aumento populacional que trouxe novas demandas a esses municípios e região geográfica a qual estão inseridos, que, em muitos casos, não ofereceram infraestrutura capaz de acompanhar esse crescimento. Logo, além de passivos ambientais ocasionados diretamente pela exploração do petróleo, há impactos ambientais e sociais que surgiram por esses fatos. Neste trabalho veremos a evolução do IDHM e o atraso em infraestrutura dos municípios sede da exploração petrolífera e seus municípios vizinhos realizando a mesma análise. A importância da produção do petróleo no cenário brasileiro pode ser vista através de sua colocação do ranking mundial dos países produtores de combustíveis fósseis e, embora seja uma atividade de alto impacto ambiental, pode, se bem administrado seus recursos, promover desenvolvimento social para as cidades onde estão localizadas essas empresas.

Palavras-chave: Meio ambiente; Impacto social; Petróleo, Exploração.
\end{abstract}

\section{PAD TECHNIQUE ON DEFORESTATION SITUATION ON PETROPOLIS' METROPOLITAN AREA - RJ IMPACT ASSESSMENT, LIABILITIES AND ENVIRONMENTAL DAMAGE IN COASTAL AREAS OF BRAZIL: COASTAL REGION NORTH FLUMINENSE}

\begin{abstract}
:
Oil exploration brought technological advances and high financial gains from direct and indirect way for municipalities to host this branch companies and workers from various fields, moving to local, State and national economy, but in this match against the advance population increase which brought new demands to these municipalities and geographical region which are inserted, which in many cases not offered infrastructure able to keep up with this growth. Soon, in addition to environmental liabilities caused directly by oil exploration, there are
\end{abstract}


environmental and social impacts that have emerged for these facts. In this work we see the development of the infrastructure and the delay IDHM municipalities oil exploration headquarters and its neighboring municipalities conducting the same analysis. The importance of oil production in the Brazilian scenario can be seen through his placing in the world ranking of the fossil fuel producing countries and, although it is an activity of high environmental impact, can, if well managed its resources, promote social development for cities where these companies are located.

Keywords: Environmental; Impact social; Petroleum; Exploration.

\section{EVALUACIÓN DEL IMPACTO, PASIVO Y EL DAÑO AMBIENTAL EN LAS ZONAS COSTERAS DE BRASIL: REGIÓN LITORAL DEL NORTE FLUMINENSE}

\section{Resumen:}

Exploración petrolera trajo avances tecnológicos y alto beneficios financieros de manera directa e indirecta para municipios albergar las empresas de esta rama y trabajadores de diversos ámbitos, mover a la economía local, estatal y nacional, pero en este partido contra la población avance aumentan que trajo nuevas exigencias a estos municipios y la región geográfica que se insertan, que en muchos casos no ofrece infraestructura capaz de continuar con este crecimiento. Pronto, además de pasivos ambientales causados directamente por exploración de petróleo, existen impactos ambientales y sociales que han surgido por estos hechos. En este trabajo podemos ver el desarrollo de la infraestructura y los municipios IDHM de retraso de aceite exploración sede y sus municipios vecinos realizando el mismo análisis. La importancia de la producción de petróleo en el escenario brasileño puede verse a través de su colocación en el ranking mundial de los países productores de combustibles fósiles y, aunque es una actividad de alto impacto ambiental, puede, si se administran bien sus recursos, promover el desarrollo social de las ciudades donde se encuentran estas empresas.

Palabras clave: Médio ambiente; Impacto social; Petróleo; Exploración.

\section{INTRODUÇÃO}

Este trabalho se baseia na Teoria Pressão-Estado-Impacto-Resposta (PEIR), proposta por Kristensen, a qual diz que as atividades humanas exercem "pressão" sobre o meio ambiente, afetando seus recursos, isto é, alterando seu "estado". Os resultados causados pela alteração de estado são compreendidos como "impacto", e a "resposta" ao "impacto" é entendida como as medidas tomadas pela sociedade para resolver ou amenizar o dano causado, e esta pode afetar desde a "pressão" até o "impacto" (KRISTENSEN, 2004).

A região do Norte Fluminense alvo deste trabalho contempla os municípios de Campos dos Goytacazes, Macaé, Quissamã e São João da Barra, inseridos no macro complexo da Bacia de Campos. Essa região tem se destacado, principalmente, pelo cultivo da cana-de-açúcar e pela exploração de petróleo (MIRANDA, SILVA e ALMEIDA, 2010, NETO e AJARA, 2006). 
O uso de agrotóxicos devido às atividades pecuárias e sucroalcooleiras na região contaminaram solos e recursos hídricos, assim como os efluentes oriundos da atividade petrolífera, reduzindo a riqueza e diversidade da biota da região (DIEGUES e ROSMAN, 1998). Relacionado à atividade petrolífera, o rápido crescimento populacional associado à falta de planejamento urbano levou a um saneamento básico precário e ao não tratamento do lixo coletado nos municípios, contribuindo para a poluição de solo e rios (SILVA et al., 2008).

O objetivo deste trabalho é avaliar a região do litoral Norte Fluminense sob a teoria PEIR, levando em consideração os ecossistemas ali presentes e as relações socioeconômicas da região, além de verificar seu potencial de gestão.

\section{METODOLOGIA}

Para poder avaliar os impactos que o uso dos ativos ambientais pela sociedade causa sobre os ecossistemas da área estudada, levou-se em conta o perfil da população, as atividades econômicas e as características geográficas pertinentes às áreas dos ativos ambientais utilizados. De forma a abranger competentemente as áreas citadas, o modelo utilizado possui os seguintes itens:

\section{Localização geográfica}

Descrição das características geográficas do litoral Norte Fluminense, tais como clima e relevo.

\section{Ecossistemas}

Descrição dos ecossistemas principais da região; das áreas protegidas; e definição das funções dos ecossistemas. principais da região; das áreas protegidas; e definição das funções dos ecossistemas, seus produtos e atributos. Para tal, é utilizada a tabela (Tabela 1) das funções, produtos e atributos dos ecossistemas brasileiros, proposta por Dugan (1990). 
Tabela 1. Funções, produtos e atributos dos ecossistemas costeiros

\begin{tabular}{|c|c|c|c|c|c|c|c|c|c|c|}
\hline \multicolumn{11}{|c|}{ Funções } \\
\hline & $\begin{array}{l}\text { Baías, estuários } \\
\text { e deltas }\end{array}$ & M angues & $\begin{array}{l}\text { Dunas e } \\
\text { falésias }\end{array}$ & $\begin{array}{l}\text { Recifes e } \\
\text { corais }\end{array}$ & $\begin{array}{l}\text { Lagunas e } \\
\text { banhados }\end{array}$ & $\begin{array}{l}\text { Planícies } \\
\text { intermarés }\end{array}$ & $\begin{array}{l}\text { Praias e } \\
\text { costões }\end{array}$ & $\begin{array}{c}\text { Ilhas e } \\
\text { arquipélagos }\end{array}$ & $\begin{array}{l}\text { Planícies } \\
\text { fluviais }\end{array}$ & $\begin{array}{l}\text { Vegetação e } \\
\text { florestas }\end{array}$ \\
\hline 1. Águas abrigadas & $\mathrm{F}$ & & & A & $\mathrm{P}$ & & & $\mathrm{P}$ & & \\
\hline 2. Águas subterrâneas & $\mathrm{A}$ & A & $\mathrm{P}$ & & $\mathrm{F}$ & A & & $\mathrm{P}$ & $\mathrm{F}$ & A \\
\hline $\begin{array}{l}\text { 3. Exportação de } \\
\text { biomassa }\end{array}$ & $\mathrm{P}$ & $\mathrm{F}$ & A & $\mathrm{F}$ & $\mathrm{F}$ & $\mathrm{F}$ & $\mathrm{P}$ & & $\mathrm{P}$ & $\mathrm{F}$ \\
\hline 4. Fonte de nutrientes & $\mathrm{P}$ & $\mathrm{F}$ & & $\mathrm{F}$ & $\mathrm{F}$ & $\mathrm{F}$ & & A & $\mathrm{F}$ & $\mathrm{F}$ \\
\hline 5. Fonte de sedimentos & $\mathrm{P}$ & A & $\mathrm{F}$ & A & & $\mathrm{F}$ & $\mathrm{F}$ & A & $\mathrm{F}$ & \\
\hline 6. Prevenção de erosão & $\mathrm{P}$ & $\mathrm{F}$ & $\mathrm{P}$ & $\mathrm{P}$ & $\mathrm{P}$ & $\mathrm{P}$ & $\mathrm{P}$ & $\mathrm{P}$ & $\mathrm{F}$ & $\mathrm{F}$ \\
\hline $\begin{array}{l}\text { 7. Prevenção de } \\
\text { inundação }\end{array}$ & $\mathrm{P}$ & $\mathrm{F}$ & A & & $\mathrm{P}$ & $\mathrm{F}$ & A & & $\mathrm{F}$ & $\mathrm{P}$ \\
\hline $\begin{array}{l}\text { 8. Proteção de } \\
\text { tempestades }\end{array}$ & $\mathrm{P}$ & $\mathrm{F}$ & $\mathrm{P}$ & $\mathrm{P}$ & $\mathrm{P}$ & A & $\mathrm{F}$ & $\mathrm{P}$ & A & \\
\hline $\begin{array}{l}\text { 9. Retenção de } \\
\text { nutrientes }\end{array}$ & $\mathrm{P}$ & $\mathrm{F}$ & & $\mathrm{F}$ & & $\mathrm{F}$ & $\mathrm{P}$ & & $\mathrm{F}$ & $\mathrm{F}$ \\
\hline $\begin{array}{l}\text { 10.. Retenção de } \\
\text { sedimentos }\end{array}$ & $\mathrm{F}$ & $\mathrm{F}$ & $\mathrm{P}$ & A & $\mathrm{F}$ & $\mathrm{F}$ & $\mathrm{P}$ & A & $\mathrm{F}$ & $\mathrm{F}$ \\
\hline 11. Via de transporte & $\mathrm{P}$ & $\mathrm{P}$ & A & A & $\mathrm{F}$ & $\mathrm{P}$ & A & $\mathrm{F}$ & $\mathrm{F}$ & \\
\hline \multicolumn{11}{|c|}{ Produtos } \\
\hline & $\begin{array}{l}\text { Baías, estuários } \\
\text { e deltas }\end{array}$ & Mangues & $\begin{array}{l}\text { Dunas e } \\
\text { falésias }\end{array}$ & $\begin{array}{c}\text { Recifes e } \\
\text { corais }\end{array}$ & $\begin{array}{l}\text { Lagunas e } \\
\text { banhados }\end{array}$ & $\begin{array}{l}\text { Planícies } \\
\text { intermarés }\end{array}$ & $\begin{array}{l}\text { Praias e } \\
\text { costões }\end{array}$ & $\begin{array}{c}\text { Ilhas e } \\
\text { arquipélagos }\end{array}$ & $\begin{array}{l}\text { Planícies } \\
\text { fluviais }\end{array}$ & $\begin{array}{l}\text { Vegetação e } \\
\text { florestas }\end{array}$ \\
\hline 1. Aquicultuira & $\mathrm{A}$ & A & & & $\mathrm{P}$ & $\mathrm{P}$ & A & & $\mathrm{P}$ & \\
\hline 2. Campos e pastagens & $\mathrm{P}$ & $\mathrm{P}$ & & & $\mathrm{P}$ & $\mathrm{F}$ & A & $\mathrm{P}$ & $\mathrm{F}$ & $\mathrm{F}$ \\
\hline 3. Espécies silvestres & $\mathrm{F}$ & $\mathrm{P}$ & $\mathrm{P}$ & & $\mathrm{P}$ & $\mathrm{F}$ & $\mathrm{P}$ & $\mathrm{P}$ & $\mathrm{F}$ & $\mathrm{F}$ \\
\hline 4. Recreação e turismo & $\mathrm{P}$ & $\mathrm{P}$ & $\mathrm{P}$ & $\mathrm{F}$ & $\mathrm{F}$ & $\mathrm{P}$ & $\mathrm{F}$ & $\mathrm{F}$ & $\mathrm{P}$ & $\mathrm{P}$ \\
\hline 5. Recursos pesqueiros & $\mathrm{F}$ & $\mathrm{F}$ & & $\mathrm{F}$ & & $\mathrm{F}$ & $\mathrm{P}$ & $\mathrm{F}$ & $\mathrm{F}$ & \\
\hline 6. Recursos agrícolas & A & A & & & $\mathrm{P}$ & $\mathrm{F}$ & A & $\mathrm{P}$ & $\mathrm{P}$ & A \\
\hline 7. Recursos minerais & $\mathrm{P}$ & A & $\mathrm{P}$ & $\mathrm{P}$ & $\mathrm{P}$ & $\mathrm{P}$ & $\mathrm{P}$ & $\mathrm{P}$ & $\mathrm{P}$ & A \\
\hline 8. Recursos florestais & A & $\mathrm{F}$ & & & & $\mathrm{P}$ & A & & A & $\mathrm{F}$ \\
\hline \multicolumn{11}{|c|}{ Atributos } \\
\hline & $\begin{array}{l}\text { Baías, estuários } \\
\text { e deltas }\end{array}$ & Mangues & $\begin{array}{l}\text { Dunas e } \\
\text { falésias }\end{array}$ & $\begin{array}{l}\text { Recifes e } \\
\text { corais }\end{array}$ & $\begin{array}{l}\text { Lagunas e } \\
\text { banhados }\end{array}$ & $\begin{array}{l}\text { Planícies } \\
\text { intermarés }\end{array}$ & $\begin{array}{l}\text { Praias e } \\
\text { costões }\end{array}$ & $\begin{array}{c}\text { Ilhas e } \\
\text { arquipélagos }\end{array}$ & $\begin{array}{l}\text { Planícies } \\
\text { fluviais }\end{array}$ & $\begin{array}{l}\text { Vegetação e } \\
\text { florestas }\end{array}$ \\
\hline 1. Diversidade biológica & $\mathrm{F}$ & $\mathrm{P}$ & & $\mathrm{F}$ & $\mathrm{F}$ & $\mathrm{F}$ & $\mathrm{P}$ & $\mathrm{F}$ & $\mathrm{P}$ & $\mathrm{F}$ \\
\hline \begin{tabular}{|l|} 
2. Diversidade e \\
patrimônio cultural
\end{tabular} & $\mathrm{P}$ & $\mathrm{P}$ & & & $\mathrm{F}$ & $\mathrm{P}$ & $\mathrm{P}$ & $\mathrm{F}$ & $\mathrm{P}$ & \\
\hline $\begin{array}{l}\text { 3. Morfologia e } \\
\text { paisagens }\end{array}$ & $\mathrm{P}$ & A & $\mathrm{F}$ & $\mathrm{F}$ & $\mathrm{P}$ & A & & $\mathrm{F}$ & A & $\mathrm{F}$ \\
\hline \multicolumn{11}{|c|}{$\mathrm{F}=$ Frequente e importante } \\
\hline
\end{tabular}

Fonte: DUGAN, 1990

De acordo com o modelo proposto por Dugan (1990), foram selecionadas as seguintes funções para cada ecossistema principal:

- Baías, estuários e deltas: 1, 4, 5, 9 e 11;

- Mangues: 3, 4, 6, 9 e 10;

- Dunas e falésias: 4, 6 e 10;

- Lagunas e banhados: 1, 4 e 9;

- Planícies intermarés: 4, 5 e 7; 
- Praias e costões: 5, 6 e 8;

- Ilhas e arquipélagos: 1, 9 e 10;

- Planícies fluviais: 2, 3 e 9;

- Vegetação e florestas: 3 e 6.

\section{População}

Com dados retirados do Sistema IBGE de Recuperação Automática, realização de análise demográfica dos municípios integrantes da região, avaliando crescimento populacional e concentração urbana.

\section{Infraestrutura}

Avaliação dos serviços de abastecimento de água, coleta de lixo e rede de esgoto na área urbana dos municípios, com base no Sistema de Informação da Atenção Básica, elaborado a partir de pesquisa de 2013 pelo Ministério da Saúde e dados do Censo de 2010 realizado pelo IBGE (IBGE, Censo 2010).

\section{Uso do solo}

Análise de como o solo utilizado, observando as atividades econômicas praticadas e os padrões de distribuição ocupacional presente.

\section{Atividades econômicas}

Detalhamento das atividades econômicas dos municípios que integram a região, considerando os ativos ambientais utilizados.

\section{RESULTADOS}

\section{Localização Geográfica}

A região do Litoral Norte Fluminense estudada se localiza no Setor 04 da Zona Costeira do Rio de Janeiro, fazendo parte do macro complexo da Bacia de Campos 
(DIEGUES e ROSMAN, 1998). Os municípios que compõem a área estudada são Campos dos Goytacazes, Macaé, Quissamã e São João da Barra, contemplando uma área total aproximada de $6.411 \mathrm{~km}^{2}$ (IBGE, 2014).

Essa região é de baixada e nela se encontra a maior lagoa de água doce do estado do Rio de Janeiro, a Lagoa Feia, que hoje possui $200 \mathrm{~km}^{2}$ de espelho d'água, $196 \mathrm{~km}^{2}$ a menos do que apontado no seu primeiro registro cartográfico realizado em 1846. O clima da região é tropical quente e úmido, com períodos de seca entre junho e agosto (DIEGUES e ROSMAN, 1998).

\section{Ecossistemas}

A área estudada compreende parte dos rios Macaé, Itabapoana e Paraíba do Sul, sendo interconectados por canais naturais e artificiais e ligados ao oceano Atlântico; e associados a manguezais, restingas, dunas e brejos de grande importância para a preservação da região, como a restinga localizada em São João da Barra, que compõe 46\% da área de restinga do Estado (DIEGUES e ROSMAN, 1998, CHEVRON/ECOLOGUS, 2006). Na área oeste da região, próxima a Serra do Mar, há floresta ombrófila densa (DIEGUES e ROSMAN, 1998). Segundo informações presentes na EIA (CHEVRON/ECOLOGUS, 2006), não foram encontrados dados que sugerem modificações das funções, produtos e atributos dos ecossistemas da região desde trabalho realizado por Diegues em meados da década de 1990. A plantação de cana-de-açúcar, a pesca artesanal e o petróleo continuam sendo os principais produtos ecossistêmicos (Neto e Ajara, 2006). Portanto, serão utilizadas as tabelas apresentadas em Diegues e Rosman (1998) referentes às funções, produtos e atributos dos ecossistemas do litoral Norte Fluminense.

Tabela 2. Funções, produtos e atributos dos ecossistemas do litoral Norte Fluminense

\begin{tabular}{|c|c|c|c|c|c|c|c|c|c|c|}
\hline $\begin{array}{c}\text { Litoral Norte } \\
\text { Fluminense }\end{array}$ & $\begin{array}{c}\text { Baías, } \\
\text { estuários } \\
\text { e deltas }\end{array}$ & Mangues & $\begin{array}{c}\text { Dunas e } \\
\text { falésias }\end{array}$ & $\begin{array}{c}\text { Recifes } \\
\text { e corais }\end{array}$ & $\begin{array}{c}\text { Lagunas e } \\
\text { banhados }\end{array}$ & $\begin{array}{c}\text { Planícies } \\
\text { intermarés }\end{array}$ & $\begin{array}{c}\text { Praias e } \\
\text { costốes }\end{array}$ & $\begin{array}{c}\text { Ilhas e } \\
\text { arquipélagos }\end{array}$ & $\begin{array}{c}\text { Planície } \\
\text { sfluviais }\end{array}$ & $\begin{array}{c}\text { Vegetação } \\
\text { e florestas }\end{array}$ \\
\hline $\begin{array}{l}\text { Funç̃es do } \\
\text { ecossistema }\end{array}$ & 5,9 & $4,6,9$ & $5,6,10$ & $\mathrm{SD}$ & $4,9,10$ & 4,5 & $5,6,8$ & $\mathrm{SD}$ & 2 & 3,6 \\
\hline $\begin{array}{l}\text { Produtos do } \\
\text { ecossistema }\end{array}$ & 5,8 & 5,8 & 4 & $\mathrm{SD}$ & 1,4 & 8 & $4,5,7$ & $\mathrm{SD}$ & $\begin{array}{c}1,2,4, \\
5,6,7\end{array}$ & 6,8 \\
\hline $\begin{array}{l}\text { Atributos do } \\
\text { ecossistema }\end{array}$ & 1,3 & 1 & 3 & $\mathrm{SD}$ & 1 & 1,2 & 3 & $\mathrm{SD}$ & $\mathrm{SD}$ & $\mathrm{SD}$ \\
\hline
\end{tabular}

Fonte: DUGAN 1990

Há 13 unidades de conservação dentro da área de estudo, divididas em 5 APAs, 3 parques, 1 reserva federal e 4 particulares (MMA). São elas: 
- APA do Sana, em Macaé;

- APA de Macaé de Cima, estadual;

- APA da Lagoa de Cima, em Campos dos Goytacazes;

- APA Waldeir Gonçalves - Serra do Itaóca, em Campos dos Goytacazes;

- APA do Morro de Santana, em Macaé;

- Parque Atalaia, em Macaé;

- Parque Natural Municipal do Estuário do Rio Macaé, em Macaé;

- Parque Estadual da Lagoa do Açu, estadual;

- Reserva Biológica União, federal;

- Reserva Particular do Patrimônio Natural Fazenda Barra do Sana, em Macaé;

- Reserva Particular do Patrimônio Natural Ponte do Baião, em Macaé;

- Reserva Particular do Patrimônio Natural Sítio Sumidouro e Sítio Peito de Pomba, em Macaé;

- Reserva Particular do Patrimônio Natural Fazenda Caruara, em São João da Barra. 
Quadro 1. Funções, produtos e atributos do ecossistema.

\begin{tabular}{|c|c|c|c|c|}
\hline \multicolumn{5}{|c|}{ Legendas } \\
\hline \multicolumn{2}{|c|}{ Funçôes do ecossistema } & \multicolumn{2}{|c|}{ Produtos do ecossistema } & \multirow{2}{*}{$\begin{array}{c}\text { Atributos do } \\
\text { ecossistema }\end{array}$} \\
\hline $\begin{array}{c}\text { 1. Águas } \\
\text { abrigadas }\end{array}$ & $\begin{array}{l}\text { 7. Prevenção } \\
\text { de inundação }\end{array}$ & 1. Agricultura & $\begin{array}{l}\text { 7. Recursos } \\
\text { minerais }\end{array}$ & \\
\hline $\begin{array}{l}\text { 2. Àguas } \\
\text { subterrâneas }\end{array}$ & $\begin{array}{l}\text { 8. Proteção de } \\
\text { tempestades }\end{array}$ & $\begin{array}{c}\text { 2. Campose } \\
\text { pastagens }\end{array}$ & $\begin{array}{l}\text { 8. Recursos } \\
\text { florestais e } \\
\text { vegetais }\end{array}$ & $\begin{array}{l}\text { 2. Diversidade e } \\
\text { patrimônio cultural }\end{array}$ \\
\hline $\begin{array}{l}\text { 3. Exportação } \\
\text { de biomassa }\end{array}$ & $\begin{array}{l}\text { 9. Retenção de } \\
\text { nutrientes }\end{array}$ & $\begin{array}{l}\text { 3. Espécies } \\
\text { silvestres }\end{array}$ & SD. Sem dados & $\begin{array}{l}\text { 3. Morfologia e } \\
\text { paisagens }\end{array}$ \\
\hline $\begin{array}{l}\text { 4. Fonte de } \\
\text { nutrientes }\end{array}$ & $\begin{array}{l}\text { 10. Retenção } \\
\text { de sedimentos }\end{array}$ & $\begin{array}{l}\text { 4. Recreação e } \\
\text { turismo }\end{array}$ & & \\
\hline $\begin{array}{l}\text { 5. Fonte de } \\
\text { sedimentos }\end{array}$ & $\begin{array}{l}\text { 11. Via de } \\
\text { transporte }\end{array}$ & $\begin{array}{l}\text { 5. Recursos } \\
\text { pesqueiros }\end{array}$ & & \\
\hline $\begin{array}{c}\text { 6. Prevenção } \\
\text { de erosão }\end{array}$ & SD. Sem dados & $\begin{array}{l}\text { 6. Recursos } \\
\text { agrícolas }\end{array}$ & & \\
\hline
\end{tabular}

\section{Impactos ambientais e sociais}

Os impactos mais notórios a população se remetem ao crescimento populacional rápido, e na contra mão, não há melhorias significativas relativas a infraestrutura urbana. Embora os municípios com maior arrecadação de royallteis investam em áreas mais nobres, existem as áreas periféricas da cidade que necessitam de mais recursos e investimentos. Outro ponto importante é a questão das cidades vizinhas que tem sua economia menos significativa, porém sofreram com aumento da densidade populacional. Esses fatores interferem diretamente no equilibrio ambiental e social daquela região.

Segundo citação de Ramos (2009), o curso do crescimento econômico no estado pressiona a fixação desordenada de grande parte da população fluminense nos municípios produtores de petróleo, ocasionando problemas nas diferentes dimensões do ecodesenvolvimento: espacial, cultural, econômica, ecológica, social e política.

\section{$\underline{\text { 3. População }}$}

No censo de 1996 pode-se observar que mais da metade da população de todos os municípios estudados habitam a área urbana. Os municípios de Macaé e Campos dos Goytacazes são majoritariamente urbanos, com percentual mínimo de $90 \%$ de habitação urbana, de acordo com o censo de 2010. Observa-se que a população de Macaé cresceu 36\% 
entre 2000 e 2010. Há um decréscimo populacional superior a 50\% em São João da Barra entre 1996 e 2000, seguido de um leve crescimento entre 2000 e 2010.

Tabela 3. Comparação populacional do litoral Norte Fluminense entre 1996 e 2010

\begin{tabular}{|l|l|l|l|l|l|l|l|}
\hline \multirow{2}{*}{$\begin{array}{l}\text { Litoral Norte } \\
\text { Fluminense }\end{array}$} & 1996 & \multicolumn{2}{l|}{2000} & 2010 \\
\cline { 2 - 8 } & Total & $\begin{array}{l}\text { Urbana } \\
\%\end{array}$ & Total & $\begin{array}{l}\text { Urbana } \\
\%\end{array}$ & Total & $\begin{array}{l}\text { Urbana } \\
\%\end{array}$ & $\begin{array}{l}\text { Densidade } \\
\left(\mathrm{Hab}^{\prime} \mathrm{km}^{2}\right)\end{array}$ \\
\hline Quissamã & 12.583 & 55,47 & 13.674 & 56,30 & 20.242 & 64,20 & 28,4 \\
\hline $\begin{array}{l}\text { São João da } \\
\text { Barra }\end{array}$ & 63.939 & 53,31 & 27.682 & 70,92 & 32.747 & 78,46 & 71,96 \\
\hline Macaé & 121.095 & 90,87 & 132.461 & 95,13 & 206.728 & 98,13 & 169,89 \\
\hline Campos & 389.547 & 85,94 & 406.989 & 89,48 & 463.731 & 90,29 & 115,16 \\
\hline
\end{tabular}

Fonte: Censo Geográfico - IBGE

As populações de Quissamã e São João da Barra são compostas principalmente por pescadores artesanais e trabalhadores na área de agropecuária, enquanto que as populações de Macaé e Campos dos Goytacazes são formadas, em sua maioria, por trabalhadores da área industrial e petroleira (DIEGUES e ROSMAN, 1998, VALPASSOS e NETO, 2006, 2006, PONCIANO et al, 2004).

\section{IDHM - Índice de Desenvolvimento Humano Municipal de Macaé e Campos dos}

\section{Goytacazes.}

Em se tratando de desenvolvimento humano, Macaé apresenta sutil, porém de significância, elevação no IDH na década entre os anos 2000 e 2010, onde em 2000 apresentava IDH 0,665 e em 2010 estava com IDH Municipal de 0.764. Na mesma linear esta a cidade de Campos dos Goytacazes que teve seu IDH no ano 2000 de 0,618 e em 2010 0,716. (IBGE Cidades http://cidades.ibge.gov.br). Foram apresentados os IDHs de Macaé e Campos dos Goytacazes tendo em vista o crescimento populacional destes municípios, em decorrência as atividades de exploração petrolífera.

Conforme publicado no Estudo Socioeconômico do TCE - RJ do ano de 2014, Macaé encontra-se na $7^{\circ}$ posição no Ranking do IDHM dos Municípios do Estado do Rio de Janeiro e Campos dos Goytacazes em $37^{\circ}$ posição. Os municípios de Quissamã e São João da Barra estão em $55^{\circ}$ lugar e $76^{\circ}$ lugar, respectivamente.

Os dados apresentados acima trazem a reflexão de alguns pensadores e pesquisadores sobre os aspectos regionais de desigualdade de diferentes, porém próximos, territórios. Onde a 
indústria petrolífera tem aumentado não só a arrecadação de divisas econômicas, mas também agravamento das mazelas sociais e ambientais.

\section{$\underline{\text { 4. Infraestrutura }}$}

O município de Quissamã é o que apresenta melhor saneamento básico dentre os quatro municípios avaliados neste trabalho; e, desde 2014, trata $100 \%$ do esgoto coletado na área urbana (HOFFMANN e CARMO, 2014). Os municípios de São João da Barra e Campos dos Goytacazes possuem majoritariamente esgoto por fossa ao invés de rede de esgoto. A coleta diária de lixo domiciliar é superior a $90 \%$ em todos os municípios.

Tabela 4. Porcentagem da rede de esgoto, água e coleta de lixo do litoral Norte Fluminense

\begin{tabular}{|l|l|l|l|}
\hline $\begin{array}{l}\text { Litoral Norte } \\
\text { Fluminense }\end{array}$ & $\begin{array}{l}\text { \% Rede } \\
\text { de esgoto }\end{array}$ & $\begin{array}{l}\% \text { Rede } \\
\text { de água }\end{array}$ & $\begin{array}{l}\% \text { Coleta } \\
\text { de lixo }\end{array}$ \\
\hline Quissamã & 74,6 & 79 & 92 \\
\hline São João da Barra & 23,7 & 69,3 & 91,6 \\
\hline Macaé & 56,7 & 68,6 & 92,4 \\
\hline Campos & 21,5 & 59,1 & 90,7 \\
\hline
\end{tabular}

\section{Fonte: MINISTÉRIO DA SAÚDE - SIBRA}

No município de Macaé, conforme Censo realizado em 2010 pelo IBGE, o abastecimento de água era feito adequadamente, através da rede geral de distribuição, em 52.765 domicílios. Formas inadequadas, como a utilização de poço ou nascente dentro ou fora da propriedade, ou o armazenamento de água da chuva, eram utilizadas em 14.125 domicílios.

O esgotamento sanitário adequado distribuía-se entre a rede geral de esgoto ou pluvial (em 45.300 domicílios) e fossa séptica (em 10.087 domicílios). Outros 11.420 utilizavam formas inadequadas como fossa rudimentar, rio, lago ou mar e valas. Não dispunham de banheiro ou sanitário 83 domicílios (IBGE Cidades http://cidades.ibge.gov.br). Esses dados demonstram que embora Macaé seja um município com grande arrecadação de impostos e royalties, ainda há muito a ser feito em critérios de infraestrutura urbana e ambiental.

\section{$\underline{\text { 5. Uso do solo }}$}

No litoral Norte Fluminense, os canaviais e a pecuária são predominantes na região. Embora o cultivo da cana-de-açúcar seja o principal produto agrícola da região, este ainda sofre com a falta de modernização dos processos de produção e colheita da cana (DIEGUES e ROSMAN, 1998, NETO e AJARA, 2006). 


\section{$\underline{6 .}$ Atividades econômicas}

Até a instalação da base de petróleo na Bacia de Campos, os municípios dessa região tinham como base econômica o cultivo de cana-de-açúcar, a criação de gado e a pesca artesanal (DIEGUES e ROSMAN, 1998, NETO e AJARA, 2006, VALPASSOS e NETO, 2006). Após o início das atividades petrolíferas na região na década de 90, nota-se um crescimento econômico nos municípios em torno da Bacia de Campos gratificados com royaltes, principalmente nos municípios de Macaé e Campos, e assim tornando o norte fluminense um importante polo petrolífero e destacando-o pela influência na economia nacional e estadual.

A Bacia de Campos é responsável por mais de $85 \%$ da produção nacional de petróleo, o que fez Macaé deixar de ser um município agropecuário para ser foco industrial de alta tecnologia. Campos dos Goytacazes, além da produção de cana-de-açúcar e da indústria alimentícia, tem um forte setor de serviços que oferece oportunidades para os demais municípios da região (NETO e AJARA, 2006).

Dado importante a ser citado é que, segundo o Anuário Estatístico Brasileiro do Petróleo, Gás Natural e Biocombustíveis 2015 da ANP - Agência Nacional de Petróleo, o Brasil esta situado na $13^{\mathrm{a}}$ posição em produção de óleo, totalizando $2,6 \%$ da produção mundial. Já na produção de gás natural, o Brasil esta na $31^{a}$ posição, totalizando $0,6 \%$ no ranking mundial.

\section{CONCLUSÃO}

Desde seu início, as atividades petrolíferas têm impulsionado o crescimento populacional e econômico dos munícipios envoltos pela Bacia de Campos, principalmente Macaé e Campos. Entretanto, os danos ambientais e sociais derivados direta e indiretamente de tais atividades contribuíram para uma maior degradação do meio ambiente, de seus ecossistemas e da população dos municípios. A fim de reduzir os impactos causados, é essencial o investimento na infraestrutura dos municípios, principalmente no que se diz respeito ao saneamento básico, e no tratamento dos efluentes das indústrias petrolíferas e sucro-alcooleiras. 


\section{REFERÊNCIAS BIBLIOGRÁFICAS}

AGÊNCIA NACIONAL DE PETRÓLEO-ANP. Anuário estatístico brasileiro de petróleo, gás e biocombustíveis 2015. Disponível em:

<http://www.anp.gov.br/?pg=78136em=et1=et2=et3=et4=ear=eps=e1458042805086>. Acesso em: 13 março de 2016.

CHEVRON/ECOLOGUS. Estudo de impacto ambiental da atividade de produção de petróleo no Campo de Frade, Bacia de Campos. Rio de Janeiro, Ecologus - Engenharia Consultiva, 2006.

DIEGUES, A.C. ; ROSMAN, P.C.C. Caracterização dos Ativos Ambientais em Áreas Selecionadas da Zona Costeira Brasileira. Ministério do Meio Ambiente Recursos Hídricos e Amazônia Legal/GERCO/PNUD. 1998.

DUGAN, P. Wetland conservation: a review of current issues and requires action. Gland: IUCN, 1990.

FUNDAÇÃO INSTITUTO BRASILEIRO DE GEOGRAFIA E ESTATÍSTICA - IBGE. Sistema IBGE de recuperação automática - SIDRA. Disponível em: 〈http://www.sidra.ibge.gov.br〉. Acesso em: 24 set. 2015.

- Área territorial brasileira $\quad 2014 . \quad$ Disponível $\quad$ em:

ftp://geoftp.ibge.gov.br/organizacao_territorial/areas_e_limites/areas_2014_xls.zip >. Acesso em: 21 mar. 2016. IBGE Cidades. Disponível em:< http://cidades.ibge.gov.br>. Acesso em 12. Mar. 2016.

HOFFMANN, S.; CARMO, C. Secretaria do ambiente divulga tabela do ICMS verde 2014. Secretaria de estado do ambiente, Rio de Janeiro, 2 abr. 2014. Disponível em: <http://www.rj.gov.br/web/sea/exibeconteudo?articleid= 2024182>. Acesso em: 25 set. 2015.

KRISTENSEN, P. The DPSIR framework. In: workshop on a comprehensive / detailed assessment of the vulnerability of water resources to environmental change in Africa using river basin approach, 2004, Nairobi, Quênia.

MINISTÉRIO DA SAÚDE. Sistema de informação da atenção básica - SIAB. Disponível em: <http://www2.datasus.gov.br/SIAB/index.php>. Acesso em: 25 set. 2015.

MINISTÉRIO DO MEIO AMBIENTE. Cadastro nacional de unidades de conservação. Disponível em: <http://www.mma.gov.br/areasprotegidas/ cadastro-nacional-de-ucs>. Acesso em: 24 set. 2015.

MIRANDA, D. S.; SILVA, R. G.; ALMEIDA, L. B. Impactos ambientais da exploração e produção de petróleo na Bacia de Campos-RJ. Bolsista de Valor: Revista de divulgação do Projeto Universidade Petrobrás e IF Fluminense, v. 1, p. 133-138, 2010.

NETO, A. F. P.; AJARA, C. Transformações recentes na dinâmica sócioespacial do Norte Fluminense. In: XV ENCONTRO NACIONAL DE ESTUDOS POPULACIONAIS, 15., 2006, Caxambú.

PONCIANO, N. J.; SOUZA P. M.; Mata, H. T. C.; VIEIRA, J. R.; MORGADO, I. F.. Análise de viabilidade econômica e de risco da fruticultura na região Norte Fluminense. Revista de economia e sociologia rural, Rio de Janeiro, v. 42, n. 4, p. 615-635, out./dez. 2004.

RAMOS, M. S. Indicadores de impacto da indústria de petróleo: Estudo de caso da região polarizada pelo município de Macaé. Dissertação de mestrado em Engenharia Ambiental, linha de pesquisa desenvolvimento e sustentabilidade, Instituto Federal de Educação, Ciência e Tecnologia Fluminense, Macaé/RJ, 2009.

SILVA, J. M. C.; BOZELLI, R.L.; SANTOS, L. F.; LOPES, A. F.. Impactos ambientais da exploração e produção de petróleo na Bacia de Campos, RJ. In: IV ENCONTRO NACIONAL DA ANPPAS, 4, 2008, Brasília.

TRIBUNAL DE CONTAS DO ESTADO DO RIO DE JANEIRO - TCE/RJ. Estudos Socioeconômicos dos Municípios do Estado do Rio de Janeiro - 2014. Secretária Geral de Planejamento, Macaé, Rio de Janeiro.

VALPASSOS, C. A. M.; NETO, J. C. D.. Diferentes percepções da natureza: as intervenções politécnicas, a fiscalização ambiental e pescadores artesanais na Lagoa Feia. Revistas Anthropológicas, ano 10, v. 17, n. 2, p. 95116, 2006. 